\title{
Emodin-8- $O$-glucuronic acid, from the traditional Chinese medicine qinghuobaiduyin, affects the secretion of inflammatory cytokines in LPS-stimulated raw 264.7 cells via HSP70
}

\author{
PING WANG ${ }^{1}$, QUANYONG HE ${ }^{2}$ and JIE $\mathrm{ZHU}^{2}$ \\ ${ }^{1}$ PET/CT Center, Hunan Provincial Tumor Hospital; ${ }^{2}$ Department of Burns and Plastic Surgery, \\ Third Xiangya Hospital, Central South University, Changsha, Hunan 410013, P.R. China
}

Received August 1, 2015; Accepted June 28, 2016

DOI: $10.3892 / \mathrm{mmr} .2016 .5512$

\begin{abstract}
Qinghuobaiduyin (QHBDY) is a traditional Chinese medicine, which has an opsonization effect on the immune system. However, which chemical compound in QHBDY underlies the therapeutic effect remains to be elucidated. The present study was designed to investigate the effect of emodin-8-O-glucuronic acid, a chemical compound isolated from QHBDY, on the secretion of inflammatory cytokines in lipopolysaccharide (LPS)-stimulated Raw 264.7 cells. The compound was isolated from QHBDY and identified as emodin-8- $O$-glucuronic acid using liquid chromatography-mass spectrometry ${ }^{\mathrm{n}}$. The results obtained from an ELISA assay showed that emodin-8-O-glucuronic acid inhibited the elevated expression levels of tumor necrosis factor- $\alpha$ (TNF- $\alpha$ ), interleukin (IL)- $1 \beta$ and IL-10 in the LPS-stimulated Raw 264.7 cells, which occurred in a dose-dependent manner. In addition, emodin-8- $O$-glucuronic acid induced the expression of heat shock protein 70 (HSP70) in the LPS-stimulated Raw 264.7 cells, as demonstrated using western blot analysis. The effect of emodin-8- $O$-glucuronic acid on the secretion of TNF- $\alpha$, IL-1 $\beta$ and IL-10 was attenuated by the knockdown of HSP70. In conclusion, the present study demonstrated that emodin-8-O-glucuronic acid effectively suppressed LPS-induced inflammatory cytokine secretion, and this effect was attained by the increased expression of HSP70.
\end{abstract}

\section{Introduction}

Qinghuobaiduyin (QHBDY) is a traditional Chinese medicine, which has been used in a clinical setting since 1995 to treat

Correspondence to: Professor Jie Zhu, Department of Burns and Plastic Surgery, Third Xiangya Hospital, Central South University, 138 Tongzipo Road, Changsha, Hunan 410013, P.R. China

E-mail: zhu_jie4145@163.com

Key words: emodin-8-O-glucuronic acid, qinghuobaiduyin, heat shock protein 70 , inflammatory cytokines burns $(1,2)$. It inhibits the expression of inflammatory mediators in the inflammatory response, and has an opsonization effect on the immune system $(3,4)$. However, which chemical compound in QHBDY underlies the therapeutic effect remains to be elucidated.

In the present study, emodin-8-O-glucuronic acid was identified as a major chemical constituent of QHBDY. The effect and mechanisms of action of emodin-8-O-glucuronic acid, which is the analogue of emodin- $8-O-\beta$-D-glucoside, on inflammatory cytokine secretion were investigated in lipopolysaccharide (LPS)-stimulated Raw 264.7 cells. The current study aimed to increase current knowledge about emodin-8-O- $\beta$-D-glucoside, and aid in the modulation of inflammatory responses.

\section{Materials and methods}

Sample extraction. QHBDY was provided by the Chinese Medicine Laboratory of The Third Xiangya Hospital of Central South University (Changsha, China). The QHBDY water decoction was concentrated to an extract and dried for $2 \mathrm{~h}$. The extract was then added to $0.5 \mathrm{ml}$ methanol and ultrasound was performed to promote mixing of the solvent and the extract. Following centrifugation at $10,000 \mathrm{x}$ g for $5 \mathrm{~min}$ at $4^{\circ} \mathrm{C}$, the supernatant was collected for analysis.

Liquid chromatography $(L C)$-mass spectrometry $(M S)^{n}$ analysis. High performance liquid chromatography (HPLC) was performed on a Hypersil GOLD column $(2.1 \times 150 \mathrm{~mm} ; 3 \mu \mathrm{m}$; Thermo Fisher Scientific, Inc., San Jose, CA, USA), using methanol and water as the mobile phase. MS was performed on an Ion Trap Mass Spectrometer (Thermo Fisher Scientific, Inc.) using electrospray ionization. The auxiliary gas flow was set to 30 units and the sheath gas flow was set to 5 units. The temperature was set to $350^{\circ} \mathrm{C}$ and the spray pressure was set at 45 psi. MS scans between 100 and $1,000 \mathrm{~m} / \mathrm{z}$ were acquired. The major chemical constituents of QHBDY were analyzed using the PubMed database (http://pubchem.ncbi.nlm.nih. gov/search/).

Cell culture and cell transfection. The RAW 264.7 cells were purchased from the American Type Culture Collection 
(Manassas, VA, USA), and cultured at $37^{\circ} \mathrm{C}$ in a $5 \% \mathrm{CO}_{2}$ atmosphere in Dulbecco's modified Eagle's medium (DMEM; Invitrogen; Thermo Fisher Scientific, Inc.) containing 10\% fetal bovine serum (Gibco; Thermo Fisher Scientific, Inc.). LPS was purchased from Sigma-Aldrich (St. Louis, MO, USA) and diluted with phosphate-buffered saline (PBS). The emodin-8-O-glucuronic acid was diluted with DMEM. The cells $\left(1 \times 10^{6}\right.$ cells/well) were treated with or without LPS $(1 \mu \mathrm{g} / \mathrm{ml})$ for $12 \mathrm{~h}$ at $37^{\circ} \mathrm{C}$ in a $5 \% \mathrm{CO}_{2}$ atmosphere in the presence of various concentrations of emodin-8-O-glucuronic acid $(0.1,1$ or $10 \mathrm{ng} / \mathrm{ml} ; 24 \mathrm{~h}$ prior to LPS treatment). Transfection of the cells ( $2 \times 10^{6}$ cells/well) with HSP70 small interfering (si) RNA was performed using Lipofectamine 2000 (Invitrogen; Thermo Fisher Scientific, Inc.), following the manufacturer's protocol.

Measurements of tumor necrosis factor- $\alpha(T N F-\alpha)$, interleukin (IL)-1 $\beta$ and IL-10. The concentrations of TNF- $\alpha$, IL-1 $\beta$ and IL-10 in the culture supernatant were determined using commercial TNF- $\alpha$, IL-1 $\beta$ and IL-10 ELISA kits (BD Biosciences, San Diego, CA, USA), according to the manufacturer's protocols.

Western blot analysis. The cells were harvested and lysed in ice-cold lysis buffer [150 mM Tris- $\mathrm{HCl}$ (pH 7.4), $150 \mathrm{mM}$ $\mathrm{NaCl}, 1 \%$ Nonidet P-40, 2 mM EDTA, $50 \mathrm{mM}$ sodium fluoride, $0.2 \%$ SDS, $100 \mathrm{mM}$ sodium vanadate and $1 \mathrm{mM}$ phenylmethyl-sulfonyl fluoride], and were then centrifuged at $150 \mathrm{x} \mathrm{g}$ for $5 \mathrm{~min}$ at $4^{\circ} \mathrm{C}$, following which the culture supernatant was collected. Protein quantification was performed using a Bradford Protein Assay kit (Beyotime Institute of Biotechnology, Shanghai, China). The proteins $(20 \mu \mathrm{g})$ were separated on a $10 \%$ SDS polyacrylamide gel, following which they were electrotransferred onto a polyvinylidene fluoride membrane (EMD Millipore, Billerica, MA, USA). Following blocking with $5 \%$ non-fat milk, the membranes were washed with Tris-buffered saline with Tween-20 3 times followed by incubation with rabbit polyclonal antibody against HSP70 (dilution 1:800; cat. no. sc-33575; Santa Cruz Biotechnology, Inc., Santa Cruz, CA, USA) and rabbit polyclonal antibody against GAPDH (dilution 1:1,000; cat. no. sc-25778; Santa Cruz Biotechnology, Inc.) overnight at $4^{\circ} \mathrm{C}$, followed by incubation with goat anti-rabbit $\operatorname{IgG}$ (dilution 1:1,000; cat. no. sc-2004; Santa Cruz Biotechnology, Inc.) for $1 \mathrm{~h}$ at $37^{\circ} \mathrm{C}$. To correct for differences in protein loading, the blots were normalized against GAPDH. The signals were visualized using an ECL western blotting kit (Pierce Biotechnology, Inc., Rockford, IL, USA).

Statistical analysis. The results are expressed as the mean \pm standard deviation and differences between two groups were assessed using Student's $t$-test. Statistical analysis was performed using SPSS software, version 19.0 (IBM SPSS, Armonk, NY, USA). $\mathrm{P}<0.05$ was considered to indicate a statistically significant difference.

\section{Results}

Identification of QHBDY compounds. LC-MS ${ }^{\mathrm{n}}$ detection was developed for the analysis of the major chemical constituents of QHBDY. By comparing with the data in the PubMed database, the major compound in the peak area at $447 \mathrm{~m} / \mathrm{z}$ was identified as emodin-8-O-glucuronic acid (Fig. 1).

Effect of emodin-8-O-glucuronic acid on the expression of inflammatory cytokines in Raw 264.7 cells. The Raw 264.7 cells were treated with LPS for $12 \mathrm{~h}$, and the expression levels of inflammatory cytokines, including TNF- $\alpha$, IL-1 $\beta$ and IL-10, were examined using ELISA assays. As shown in Fig. 2, the expression levels of TNF- $\alpha$, IL- $1 \beta$ and IL-10 were significantly increased in the supernatant of cells following treatment with LPS, compared with the levels following treatment with PBS.

The LPS-stimulated Raw 264.7 cells were then treated with various concentrations of emodin-8-O-glucuronic acid for $24 \mathrm{~h}$, following which the expression levels of TNF- $\alpha$, IL-1 $\beta$ and IL-10 were measured. The results from the ELISA assays revealed that the elevated expression levels of TNF- $\alpha$, IL-1 $\beta$ and IL-10 in the LPS-stimulated Raw 264.7 cells were suppressed by emodin-8-O-glucuronic acid, and this occurred in a dose-dependent manner. Treatment with $10 \mathrm{ng} / \mathrm{ml}$ emodin-8-O-glucuronic acid resulted in a marked inhibitory effect on the expression levels of TNF- $\alpha$, IL-1 $\beta$ and IL-10.

Effect of emodin-8-O-glucuronic acid on the expression of HSP70 in Raw 264.7 cells. The expression of HSP70 in Raw 264.7 cells treated with LPS were examined using western blot analysis. The results revealed that, compared with the cells in the control group, no significant alterations in the expression of HSP70 were identified in the Raw 264.7 cells treated with LPS.

The present study also examined the expression levels of HSP70 in LPS-stimulated Raw 264.7 cells following treatment with various concentrations of emodin-8-O-glucuronic acid for $24 \mathrm{~h}$. It was shown that emodin-8- $O$-glucuronic acid significantly increased the expression of HSP70, in a dose-dependent manner, with the maximum effect at $10 \mathrm{ng} / \mathrm{ml}$ (Fig. 3).

HSP70 knockdown attenuates the effect of emodin-8-O-glucuronic acid on the expression of inflammatory cytokines. To investigate whether HSP70 mediates the effect of emodin-8-O-glucuronic acid on the expression levels of TNF- $\alpha$, IL-1 $\beta$ and IL-10, HSP70 siRNA was transfected into the LPS-stimulated Raw 264.7 cells to knock down endogenous HSP70. As shown in Fig. 4, the increased expression of HSP70 observed following treatment with $10 \mathrm{ng} / \mathrm{ml}$ emodin-8-O-glucuronic acid was significantly decreased in the cells transfected with HSP70 siRNA.

Furthermore, the results revealed that HSP70 knockdown eliminated the decreased expression levels of TNF- $\alpha$, IL-1 $\beta$ and IL-10 induced by emodin-8- $O$-glucuronic acid treatment in the LPS-stimulated Raw 264.7 cells (Fig. 5).

\section{Discussion}

Emodin-8-O- $\beta$-D-glucoside was first found as a component of the traditional Chinese medicinal herb, Polygonum cuspidatum Sieb. et Zucc (5). Xiang et al (6) reported that the chemical compound, emodin-8-O- $\beta$-D-glucoside can also be isolated from the herb Polygonum amplexicaule D. 
A

RT: 39.81 AV: 1 NL: $6.58 \mathrm{E} 7$

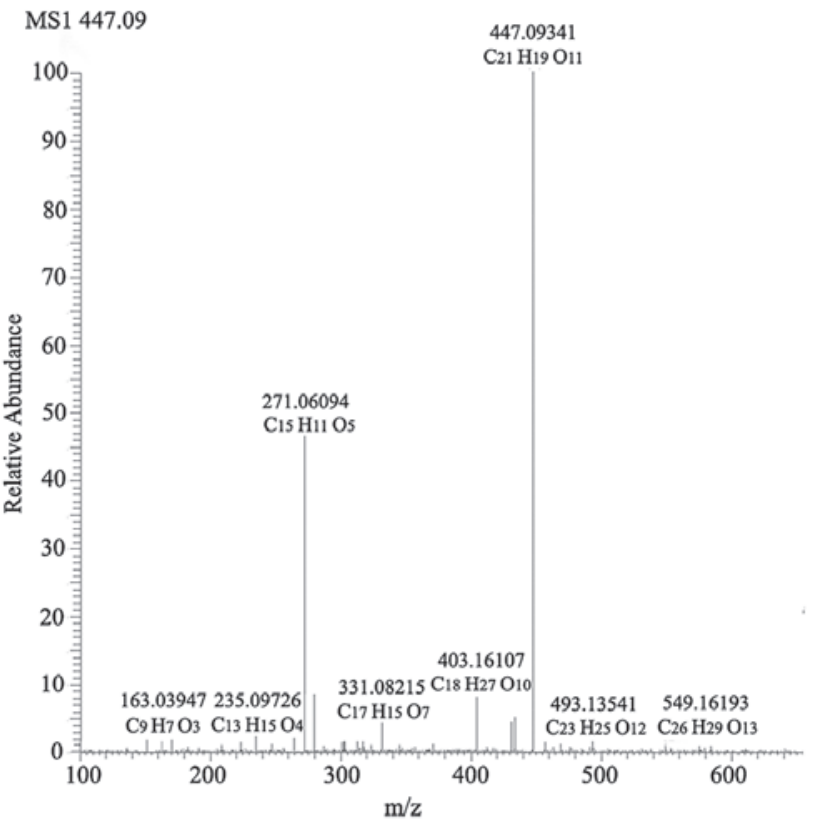

C

RT: 39.67-39.75 AV: 3 NL: 9.64E6

T: Average spectrum MS3 447.13, 271.06

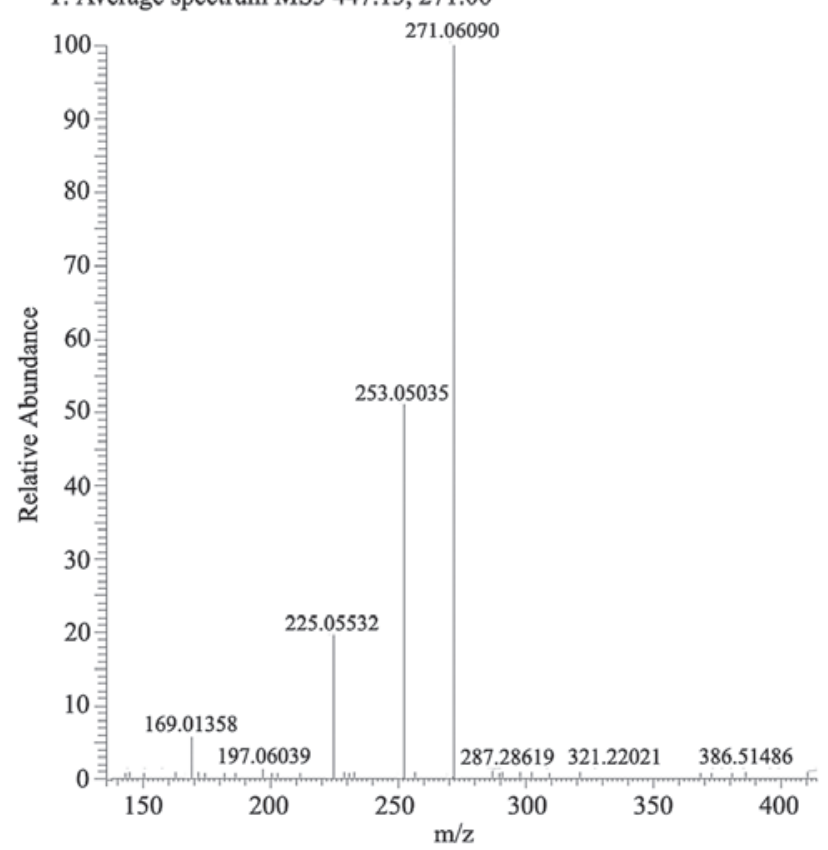

D
B

RT: 38.35-39.74 AV: 6 NL:1.17E8

T: Average spectrum MS2 447.13

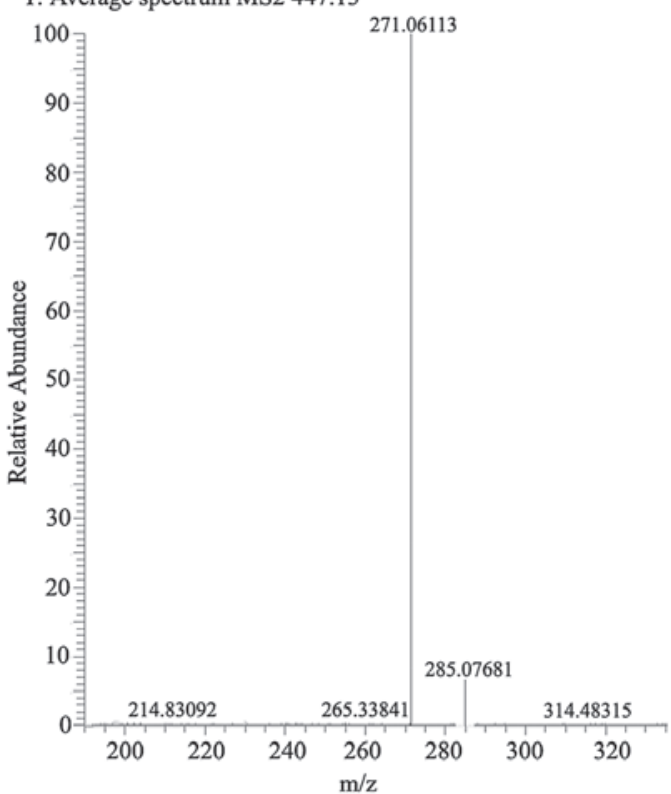<smiles>Cc1cc(O)c2c(c1)C(=O)c1cc(O)cc(OC3O[C@H](C(=O)O)C(O)[C@H](O)C3[OH+])c1C2=O</smiles>

Figure 1. LC-MS ${ }^{\mathrm{n}}$ analysis and the structure of emodin-8- $O$-glucuronic acid. (A-C) Results of the LC-MS ${ }^{\mathrm{n}}$ analysis of emodin-8- $O$-glucuronic acid. (A) MS1, (B) MS2 and (C) MS3 spectra of emodin-8- $O$-glucuronic acid. (D) Chemical structure of emodin-8- $O$-glucuronic acid. LC-MS, liquid chromatography-mass spectrometry.

Don var. sinense Forb. (Polygonaceae). In the present study, it was revealed that emodin-8-O-glucuronic acid is a major component of QHBDY, and is the analogue of emodin-8-O- $\beta$-D-glucoside.

Emodin-8- $O-\beta$-D-glucoside exerts antioxidative effects and is widely used to treat acute hepatitis (7). A study by Wang et al (7) demonstrated that emodin- $8-O-\beta$-D-glucoside is able to provide neuroprotection against the focal cerebral injury induced by ischemia and reperfusion, and inhibit glutamate neurotoxicity through antioxidative mechanisms.
Previously, it was found that emodin-8-O- $\beta$-D-glucoside directly stimulates the proliferation and differentiation of osteoblasts, thus promoting the healing of bone injury (6). Until now, there have been no reports regarding the potential effect of emodin-8-O- $\beta$-D-glucoside on immune reactions. Therefore, the present study performed in vitro experiments to investigate the functions of emodin-8- $O$-glucuronic acid.

TNF- $\alpha$ and IL- $1 \beta$ are key pro-inflammatory cytokines, and IL-10 is an anti-inflammatory cytokine, all of which are produced by inflammatory cells and are vital in the 


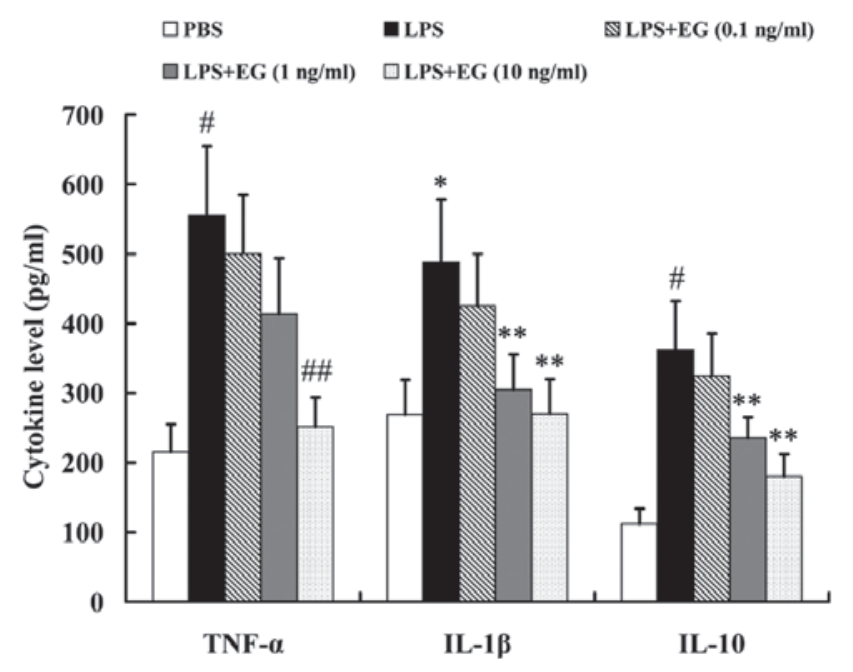

Figure 2. Effect of emodin-8- $O$-glucuronic acid on the expression of inflammatory cytokines in Raw 264.7 cells. " $\mathrm{P}<0.05$ and ${ }^{\text {}} \mathrm{P}<0.01$, compared with the PBS group; ${ }^{* *} \mathrm{P}<0.05$ and ${ }^{\# \#} \mathrm{P}<0.01$, compared with the LPS group. PBS, phosphate-buffered saline; LPS, lipopolysaccharide; EG, emodin-8- $O$-glucuronic acid; TNF, tumor necrosis factor; IL, interleukin.

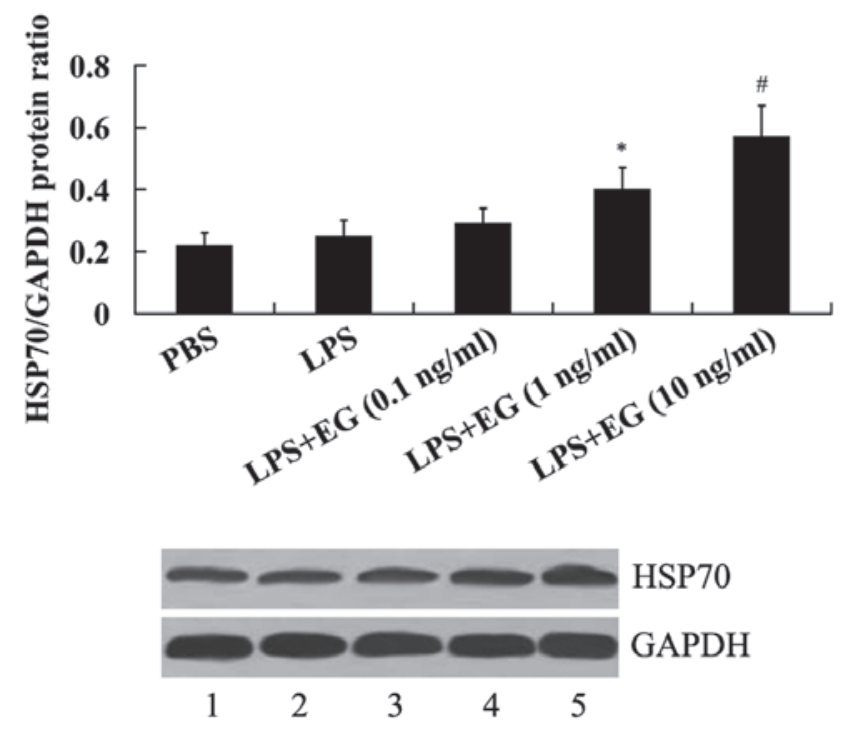

Figure 3. Effect of emodin-8- $O$-glucuronic acid on the expression of HSP70 in Raw 264.7 cells. Lane 1, PBS; lane 2, LPS; lane 3, LPS+EG $(0.1 \mathrm{ng} / \mathrm{ml})$; lane 4, LPS+EG (1 ng/ml); lane 5, LPS+EG (10 ng/ml). "P<0.05 and " $\mathrm{P}<0.01$, compared with the LPS group. PBS, phosphate-buffered saline; LBS, lipopolysaccharides; EG, emodin-8- $O$-glucuronic acid; HSP70, heat shock protein 70

inflammatory response (8-12). The abnormal production of these cytokines has been implicated in the pathogenesis of inflammation (13-16). LPSs are potent stimulators of inflammatory reactions $(17,18)$. Investigating the changes in the expression of TNF- $\alpha$, IL-1 $\beta$ and IL-10 may improve understanding of the effect of emodin-8-O- $\beta$-D-glucoside on inflammatory reactions. The present study demonstrated that emodin-8-O-glucuronic acid exerted inhibitory effects on the secretion of TNF- $\alpha$, IL-1 $\beta$ and IL-10 in LPS-stimulated Raw 264.7 cells in a dose-dependent manner.

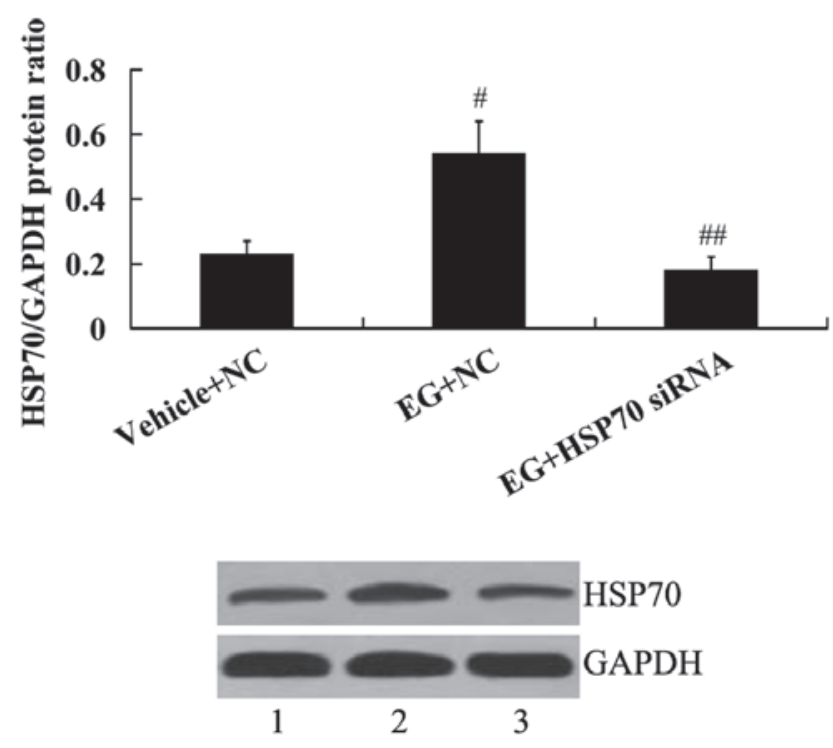

Figure 4. Expression of HSP70 in LPS-stimulated Raw 264.7 cells following transfection with HSP70 siRNA. Lane 1, Vehicle+NC; lane 2, EG+NC; lane 3, EG+HSP70 siRNA. "P<0.01 compared with the Vehicle+NC; ${ }^{\# \#} \mathrm{P}<0.01$ compared with the EG+NC. LPS, lipopolysaccharide; EG, emodin-8- $O$-glucuronic acid; HSP70, heat shock protein 70; NC, negative control; siRNA, small interfering RNA.

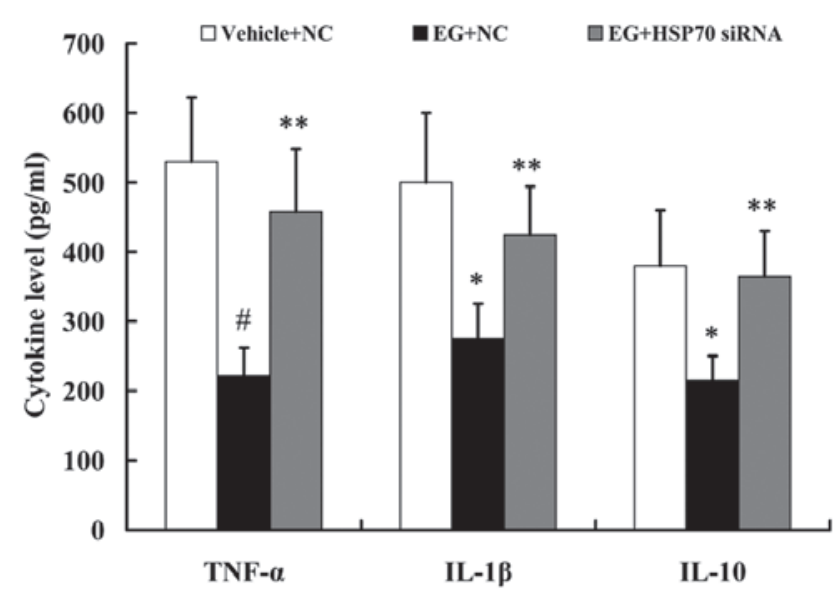

Figure 5. HSP70 knockdown attenuates the effect of emodin-8- $O$-glucuronic acid on the expression of inflammatory cytokines. " $\mathrm{P}<0.05$ and ${ }^{*} \mathrm{P}<0.01$, compared with the Vehicle+NC group; ${ }^{* *} \mathrm{P}<0.05$, compared with the EG+NC group. EG, emodin-8- $O$-glucuronic acid; $\mathrm{NC}$, negative control; HSP70, heat shock protein70; TNF, tumor necrosis factor; IL, interleukin; siRNA, small interfering RNA.

HSP70 is an ATPase, which protects against a variety of stresses, thus regulating multicellular physiological functions (18-21). It has been demonstrated that HSP70 is important in modulating the febrile response to LPS at the cell level and the whole organism level $(22,23)$. The upregulation of HSP70 represses LPS-induced cytokine expression in animals and cultured macrophages (24-26). In our previous study (27), it was demonstrated that QHBDY induced the expression of HSP70 in a severe burn model. In the present study, the effect of emodin-8- $O$-glucuronic acid on the expression of HSP70 was investigated, and the findings suggested 
that emodin-8-O-glucuronic acid induced the expression of HSP70 in the LPS-stimulated Raw 264.7 cells. Furthermore, it was found that the effect of emodin-8-O-glucuronic acid on the secretion of inflammatory cytokines was attenuated by the knockdown of HSP70, indicating that the effect of emodin-8-O-glucuronic acid was established through HSP70.

In conclusion, the present study provided the first evidence, to the best of our knowledge, that emodin-8-O-glucuronic acid effectively suppressed the LPS-induced secretion of TNF- $\alpha$, IL-1 $\beta$ and IL-10 in Raw 264.7 cells, and that the mechanism underlying this effect was mediated by HSP70. These findings expand on current knowledge regarding QHBDY and emodin-8-O-glucuronic acid, and reveal a molecular mechanism underlying the opsonization effect of the traditional Chinese medicine.

\section{References}

1. Muñoz J, Albillos A, Pérez-Páramo M, Rossi I and Alvarez-Mon M: Factors mediating the hemodynamic effects of tumor necrosis factor-alpha in portal hypertensive rats. Am J Physiol 276: G687-G693, 1999.

2. Suemasu S, Tanaka K, Namba T, Ishihara T, Katsu T, Fujimoto M, Adachi H, Sobue G, Takeuchi K, Nakai A and Mizushima T: A role for HSP70 in protecting against indomethacin-induced gastric lesions. J Biol Chem 284: 19705-19715, 2009.

3. Bromberg Z, Raj N, Goloubinoff P, Deutschman CS and Weiss YG: Enhanced expression of 70-kilodalton heat shock protein limits cell division in a sepsis-induced model of acute respiratory distress syndrome. Crit Care Med 36: 246-255, 2008.

4. Wilmore DW and Robinson MK: Metabolism and nutrition support. In: Surgical Basic Science. Fischer JE and Holmes CR (eds). 7th Edition. Mosby Press Co., St. Louis, MO, p125, 1993.

5. Yi T, Zhang H and Cai Z: Analysis of Rhizoma Polygoni Cuspidati by HPLC and HPLC-ESI/MS. Phytochem Anal 18: 387-392, 2007

6. Xiang $\mathrm{MX}, \mathrm{Xu} \mathrm{Z}, \mathrm{Su} \mathrm{HW}, \mathrm{Hu} \mathrm{J}$ and Yan $\mathrm{YJ}$ : Emodin-8-O- $\beta$-D-glucoside from Polygonum amplexicaule D. Don var. sinense Forb. promotes proliferation and differentiation of osteoblastic MC3T3-E1 cells. Molecules 16: 728-737, 2011.

7. Wang C, Zhang D, Ma H and Liu J: Neuroprotective effects of emodin-8-O-beta-D-glucoside in vivo and in vitro. Eur J Pharmacol 577: 58-63, 2007.

8. Shurety W, Merino-Trigo A, Brown D, Hume DA and Stow JL: Localization and post-Golgi trafficking of tumor necrosis factor-alpha in macrophages. J Interferon Cytokine Res 20: 427-438, 2000

9. Woolley DE and Tetlow LC: Mast cell activation and its relation to proinflammatory cytokine production in the rheumatoid lesion. Arthritis Res 2: 65-74, 2000.

10. Vigil KJ, Adachi JA and Chemaly RF: Viral pneumonias in immunocompromised adult hosts. J Intensive Care Med 25: $307-326,2010$.
11. Lilja I, Gustafson-Svärd C, Franzén L and Sjödahl R: Tumor necrosis factor-alpha in ileal mast cells in patients with Crohn's disease. Digestion 61: 68-76, 2000.

12. Wei H, Yin L, Feng S, Wang X, Yang K, Zhang A and Zhou H: Dual-parallel inhibition of IL-10 and TGF- $\beta 1$ controls LPS-induced inflammatory response via NF- $\mathrm{BB}$ signaling in grass carp monocytes/macrophages. Fish Shellfish Immunol 44: 445-452, 2015.

13. Dombrovskiy VY, Martin AA, Sunderram J and Paz HL: Rapid increase in hospitalization and mortality rates for severe sepsis in the United States: A trend analysis from 1993 to 2003. Crit Care Med 35: 1244-1250, 2007.

14. Hlava N, Niemann CU, Gropper MA and Melcher ML: Postoperative infectious complications of abdominal solid organ transplantation. J Intensive Care Med 24: 3-17, 2009.

15. Kim KD, Zhao J, Auh S, Yang X, Du P, Tang H and Fu YX: Adaptive immune cells temper initial innate responses. Nat Med 13: 1248-1252, 2007.

16. Zhao J, Yang X, Auh SL, Kim KD, Tang H and Fu YX: Do adaptive immune cells suppress or activate innate immunity? Trends Immunol 30: 8-12, 2009.

17. Wynn J, Cornell TT, Wong HR, Shanley TP and Wheeler DS: The host response to sepsis and developmental impact. Pediatrics 125: 1031-1041, 2010.

18. Unuma K, Aki T, Funakoshi T, Yoshida K and Uemura K: Cobalt protoporphyrin accelerates TFEB activation and lysosome reformation during LPS-induced septic insults in the rat heart. PLoS One 8: e56526, 2013.

19. Clerico EM, Tilitsky JM, Meng W and Gierasch LM: How hsp70 molecular machines interact with their substrates to mediate diverse physiological functions. J Mol Biol 427: 1575-1588, 2015.

20. Kregel KC: Heat shock proteins: Modifying factors in physiological stress responses and acquired thermotolerance. J Appl Physiol (1985) 92: 2177-2186, 2002.

21. Mallouk Y, Vayssier-Taussat M, Bonventre JV and Polla BS: Heat shock protein 70 and ATP as partners in cell homeostasis (Review). Int J Mol Med 4: 463-474, 1999.

22. Kluger MJ, Rudolph K, Soszynski D, Conn CA, Leon LR, Kozak W, Wallen ES and Moseley PL: Effect of heat stress on LPS-induced fever and tumor necrosis factor. Am J Physiol 273: R858-R863, 1997.

23. Dokladny K, Kozak A, Wachulec M, Wallen ES, Menache MG, Kozak W, Kluger MJ and Moseley PL: Effect of heat stress on LPS-induced febrile response in D-galactosamine-sensitized rats. Am J Physiol Regul Integr Comp Physiol 280: R338-R344, 2001.

24. Dokladny K, Lobb R, Wharton W, Ma TY and Moseley PL: LPS-induced cytokine levels are repressed by elevated expression of HSP70 in rats: Possible role of NF-kappaB. Cell Stress Chaperones 15: 153-163, 2010.

25. Snyder YM, Guthrie L, Evans GF and Zuckerman SH: Transcriptional inhibition of endotoxin-induced monokine synthesis following heat shock in murine peritoneal macrophages. J Leukoc Biol 51: 181-187, 1992.

26. Hagiwara S, Iwasaka H, Matsumoto S and Noguchi T: Changes in cell culture temperature alter release of inflammatory mediators in murine macrophagic RAW264.7 cells. Inflamm Res 56: 297-303, 2007.

27. Zhu J, Wang P, He Q, Zhou J and Luo C: Evidence of an anti-apoptotic effect of qinghuobaiduyin on intestinal mucosa following burn injury. Exp Ther Med 6: 1390-1396, 2013. 